Research Article

\title{
Remote Fault-Tolerant Control for Industrial Smart Surveillance System
}

\author{
Atif Mahmood (D), ${ }^{1}$ Abdul Qayyum Khan, ${ }^{1}$ Ghulam Mustafa, ${ }^{1}$ Nasim Ullah, \\ Muhammad Abid, ${ }^{1}$ and Aadil Sarwar Khan ${ }^{1}$ \\ ${ }^{1}$ Department of Electrical Engineering, Pakistan Institute of Engineering and Applied Sciences, Islamabad, Pakistan \\ ${ }^{2}$ Department of Electrical Engineering, College of Engineering, Taif University KSA, P.O. Box 11099, Taif 21944, Saudi Arabia \\ Correspondence should be addressed to Atif Mahmood; engg.khaki@gmail.com
}

Received 14 March 2021; Revised 12 May 2021; Accepted 24 May 2021; Published 10 June 2021

Academic Editor: Oh-Min Kwon

Copyright ( 2021 Atif Mahmood et al. This is an open access article distributed under the Creative Commons Attribution License, which permits unrestricted use, distribution, and reproduction in any medium, provided the original work is properly cited.

We design a remote fault-tolerant control for an industrial surveillance system. The designed controller simultaneously tolerates the effects of local faults of a node, the propagated undesired effects of neighboring connected nodes, and the effects of networkinduced uncertainties from a remote location. The uncertain network-induced time delays of communication links from the sensor to the controller and from the controller to the actuator are modeled using two separate Markov chains and packet dropouts using the Bernoulli process. Based on linear matrix inequalities, we derive sufficient conditions for output feedbackbased control law, such that the controller does not directly depend on output, for stochastic stability of the system. The simulation study shows the effectiveness of the proposed approach.

\section{Introduction}

Surveillance of processes is generally defined as monitoring of activities and behavior to collect information to influence, manage, or direct. In industries, the surveillance is done via industrial drones that have applications in defense, newsgathering, telecommunication, humanitarian needs, public and private safety, traffic monitoring, security, and process industry [1]. These drones are equipped with a gimbal camera sensor that collects information and sends it to a remote control room through a communication network. A smart surveillance system makes use of a remote control mechanism that can handle the surveillance operation. The remote control of geographically distributed networked systems is widely used [2-4] for cost reduction, simple installation, and maintenance and for increasing efficiency and reliability in the recent industrial revolution. Recent advances in industrial control mechanisms call for more sophisticated schemes for remote surveillance. Such surveillance schemes are desired to take care of detecting and diagnosing faults and tolerating them if needed.
In controlling plants from a remote location over communication networks, controllers face two main issues that are network-induced uncertainties and faults. The network lines induce random time delays and data packet dropouts during plant-controller communication over a network. These communication imperfections degrade the system performance. To achieve a prescribed performance, control engineers are trying to tolerate its effects. For this purpose, the researchers modeled the time delays as constant, time-varying, or random variable $[5,6]$. Markov chain is suitable to model for these network-induced effects as it depends on the current state only. However, in practice, especially in NCSs, the exact values of Transition Probabilities (TPs) are either hard to determine or costly. Consequently, for a practical design, it is necessary to consider more complex TPs, such as uncertain TPs or partly unknown TPs [7-9]. Similarly, packet dropouts are modeled with probability-based switches for networked systems [10-13].

Parallel with network uncertainties faults in the sensor, the actuator, or in the process itself may occur in networked 
systems. A lot of work has been done in the fault diagnosis and tolerance of such industrial processes [14] as maintenance and repair cannot always be achieved immediately in automated systems. For remotely distributed connected systems, these faults become more uncertain due to the propagation of fault over network links to neighboring connected nodes [15]. The propagated faulty effects may be nonlinear that may add to the already existing faults of other connected nodes [16-18]. The objective is to tolerate the effects of local simultaneous faults, propagated effects of faults that occur at other connected nodes, and networkinduced uncertainties.

Researchers have put their efforts into the diagnosis of faults in such geographically distributed connected systems $[19,20]$. Most of the researchers addressed either the actuator faults or sensor faults with network-induced effects especially with time delays [14]. In real-time applications, both the sensor and actuator faults may occur simultaneously. That is why it is important to address both types of faults at a time. For this purpose, the authors of [21] addressed both sensor and actuator simultaneous faults but with relatively conservative fault models. The authors' designed a state feedback-based controller that has limitations in practical applications due to the rare accessibility of fully known state vectors. One other source of motivation is that the existing literature addressed the components' faults only without the propagated effects [15-17] of faults of other nodes. The authors of [18] presented a new fault model for actuators and tolerated its effects using a state feedback controller. The model addressed the nonlinear effects too that may occur due to faults or other technical limitations. In this approach, the following things are important to note. Firstly, we need tolerance of simultaneous sensors and actuators faults in practical applications while the authors addressed only actuator faults. Secondly, the designed state feedback controller has limitations from a practical point of view due to the rare accessibility to all states. The third one is that only time delays are considered in the sensor-to-controller link that means the controller receives packets over a link and command the actuator directly without network link. Furthermore, packet dropout, which may occur in links due to network traffic, is not taken into account in the same link.

To address the aforementioned issues, we have designed a remote fault-tolerant control for an industrial smart surveillance system with the following contributions.

(1) The random time delay and the data packet dropouts are modeled in sensor-to-controller (S/C) links and controller-to-actuator (C/A) links separately. The time delays are modeled using two separate Markov chains with partly unknown transition probabilities and packet dropouts with two separate Bernoulli processes. The formulation helps us to establish a new framework to analyze a remote fault-tolerant control problem for industrial systems over a network.

(2) The mode-dependent output feedback-based remote controller is designed such that the controller does not directly depend on the sensor's output. Such dynamic remote fault-tolerant controllers are significant for industrial processes where the system's states are unknown.

(3) Sufficient conditions are derived in terms of linear matrix inequality (LMI) for a closed-loop Markovian Jump nonlinear system. The derived control law ensures the stochastic stability of the system and tolerates the sensors and actuator simultaneous faults to maintain the prescribed performance in a networked environment.

We organized the subsequent sections of the paper in the following way. We dedicate Section 2 for system description and problem formulation, where uncertain network-induced imperfections are modeled as a Markov chain, and then present a new fault model for networked systems. The symbols used throughout the paper are given in Table 1. In Section 3, remote fault-tolerant control is designed for the industrial surveillance system using a new LMI. In Section 4, simulation results are presented and compared with a conventional remote control for the effectiveness of our proposed control design, and then, the paper is concluded.

\section{System's Description and Problem Formulation}

2.1. Smart Surveillance System. The smart surveillance system consists of multiple drones connected via a shared communication network. Each drone is equipped with a gimbal camera sensor and is controlled from a remote location, as shown in Figure 1. The drone is used to carry the gimbal-based camera sensor to different locations for monitoring and surveillance. The collected information via a camera sensor or from other connected drones is then sent to a remote control room. The camera sensor is pivoted in the inner channel of the gimbal system that decouples it from the drone body. The decoupling helps the camera sensor to collect information with minimum viewpoint changes [22]. The camera sensor inside the gimbal system is actuated using BLDC motors according to the commands of the controller. This gimbal-based smart surveillance system for industries has to be controlled from remote locations for safe and easy use.

To make the smart monitoring and surveillance system, we consider two things. Firstly, the controller can stabilize and control the camera system from a remote location for safe and easy use. Secondly, the designed controller can tolerate the effects of local faults, propagated or external faults that may be nonlinear, and network-induced uncertainties simultaneously. The local faults are sensors and actuators faults in our case. This smart surveillance system has applications in industries where we need information from remote locations with safety and ease.

2.2. Problem Formulation. Consider the remote control scenario of a smart surveillance system in Figure 2 where a remote controller controls the gimbal camera sensor 
TABle 1: Notations with description used throughout the manuscript.

\begin{tabular}{lc}
\hline \multicolumn{3}{c}{ Notations used for mathematical modeling } \\
Parameter & Description \\
\hline$\tau_{k}$ & Loop delay \\
$\tau_{k s c}$ & Time delay from the sensor to the controller \\
$\tau_{k c a}$ & Time delay from the controller to the actuator \\
$u(k)$ & Controller's output \\
$u^{*}(k)$ & Actuator's output with faults and network effects \\
$y(k)$ & Sensor's output \\
$y^{*}(k)$ & Controller's input with faults and network effects \\
$\Theta$ & Actuator fault matrix \\
$\Theta_{1}$ & Markov chain for time delays in sensor-to-controller \\
$\Theta_{2}$ & Markov chain for time delays in controller-to- \\
$*$ & actuator links \\
$\operatorname{Pr}(\cdot)$ & Symmetric entries \\
$\Theta^{T} P(\ldots)$ & Probabilities \\
\hline
\end{tabular}

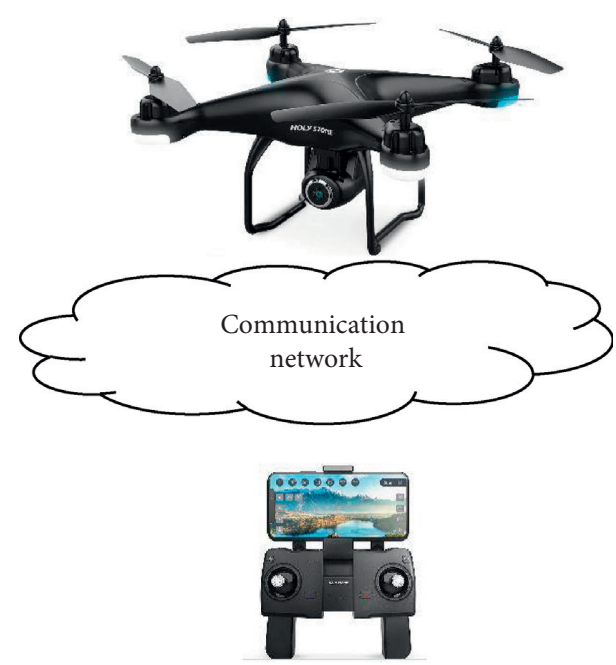

Figure 1: Remote control of the industry surveillance system.

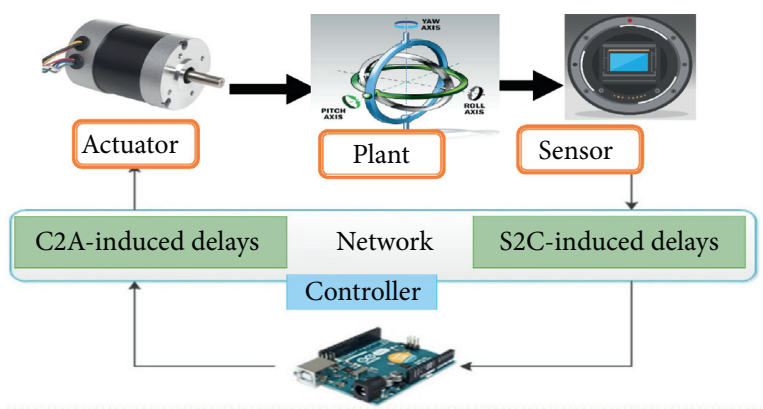

FIgure 2: Control over network.

through a shared communication network. The system modeling with related detail can be seen in $[23,24]$. The state dynamics of the system are given as

$$
\begin{aligned}
x(k+1) & =A x(k)+B u^{*}(k), \\
y(k) & =C x(k),
\end{aligned}
$$

where $x(k) \in \mathbb{R}^{n}$ is the state vector, $u^{*}(k) \in \mathbb{R}^{m}$ is the input signal, and $y(k) \in \mathbb{R}^{p}$ is the output. $A, B$, and $C$ are system constant matrices of compatible dimensions. Besides, the matrix $C$ has a full-row rank.

2.3. Sensor-to-Controller Communication. From the sensor to the controller, the output signals propagate via network links and then are available to the controller. Output of the camera sensor available to the controller may be with faulty effects in the camera sensor and with communication uncertainties. To model the output $y(k)$ with faults, we introduce

$$
y^{*}(k)=\Omega y(k)+f(\cdot) .
$$

The sensors faults are modeled as

$$
\Omega=\operatorname{diag}\left(\omega_{1}, \omega_{2}, \ldots, \omega_{p}\right),
$$

where $\Omega$ denotes the faults' matrix and the functions $\omega_{l} \in[0,1]$, for $l=1, \ldots, p$, indicate the status of each individual actuator as

$$
\omega_{l}= \begin{cases}1, & \text { the sensor is working normally, } \\ 0, & \text { the sensor is failed completely, } \\ \text { otherwise, } & \text { the sensor is partially working. }\end{cases}
$$

For the gimbal-based surveillance system, $p=1$ because of only one camera sensor that is in the inner channel of the gimbal system. $f(\cdot)$ is a nonlinear term that may add due to occurrence of fault or other technical limitation [18].

The aforementioned faulty output data may or may not reach to the controller due to network traffic. The scenario can be modeled with random events as

Event 1: Succesful transmission and output is available,

Event 2: Link fail and output is not available.

To model the random events, we introduce $a(k)$ which is a binary random vector. A value " 1 " of $a_{i}(k)$ indicates that the packet was delivered successfully in the $i_{\text {th }}$ channel and "0" indicates that the packet was dropped. Here, $i \in\{1,2, \ldots, m\}$. We assume the following probabilities for $a_{i}(k)$ :

$$
\begin{aligned}
& \operatorname{Pr}\left(a_{i}(k)=1\right)=E\left[a_{i}(k)\right]=\bar{a}_{i}(k), \\
& \operatorname{Pr}\left(a_{i}(k)=0\right)=1-\bar{a}_{i}(k),
\end{aligned}
$$

where $\operatorname{Pr}\left\{a_{i}(k)=1\right\}$ is the probability of packet transmission in $S / C$ links and $E$ is the mathematical expectation. When the probability of $a_{i}(k)=1$, it means maximum probability or successful transmission of data packet through S/C links, else the packet is lost. 
The data packets that reach from the sensor to the controller face random time delays $\tau_{k s c}$. We assume that $\tau_{k s c}$ varies randomly between 0 and $\tau_{1}$, where $\tau_{1}$ is the maximum time delay of a packet. We model the time delays $\tau_{k s c}$ with a Markov chain $\Theta_{1}$ with $N$ number of stats, i.e., $\Theta_{1} \in\left\{1,2, \ldots, N_{1}\right\}$. Let $\rho_{1}$ be the transition probability matrix of the Markov chain that is given as

$$
\rho_{1}=\left[\rho_{i j}\right]
$$

where $\quad \rho_{i j}=\mathbb{P}\left(\Theta_{1}=j \mid \Theta_{1}=i\right)=\mathbb{P}\left(\tau_{k s c+1}=j \mid \tau_{k s c}=i\right) \geq 0$, for all $i, j \in \Gamma_{1}$ and $\Sigma_{j=0}^{\tau_{1}} \rho_{i j}=1, \rho_{i j} \geq 0$. For example, if $\tau_{1}=3$, the complex transition probability matrix $\rho$ is given as

$$
\rho=\left[\begin{array}{cccc}
\rho_{00} & \rho_{01} & ? & \rho_{03} \\
? & \rho_{11} & \rho_{12} & ? \\
\rho_{20} & \rho_{21} & ? & \rho_{23} \\
\rho_{30} & ? & \rho_{32} & ?
\end{array}\right],
$$

where ? in the matrix are unknown state transition probabilities of time delays and the rest of entries are known transition probabilities.

The stochastic faulty output that is available to the controller is

$$
y^{*}(k)=\Omega \bar{a}_{i}(k) C x\left(k-\tau_{k s c}\right)+f(\cdot) .
$$

Due to the network-induced probabilistic packet dropouts and time delays in the network links, the controller mode may not synchronize with the system modes. For this networked system, we introduce a dynamic output feedbackbased remote control $F\left(\tau_{k s c}\right)$ as follows:

$$
\begin{aligned}
& u(k)=F\left(k-\tau_{k s c}\right) y^{*}(k), \\
& u(k)=F\left(k-\tau_{k s c}\right)\left[\Omega \bar{a}_{i}(k) C x\left(k-\tau_{k s c}\right)+f(\cdot)\right] .
\end{aligned}
$$

Remark 1. The direct output of the camera sensor is not available to the controller due to network impurities. The network uncertainties are stochastic, that is why the output may or may not be available. The available output is the delayed version with a time delay of $\tau_{k s c}$ and maybe with the effects of faults.

2.4. Controller-to-Actuator Communication. The controller output $u(k)$ propagates via network links from the controller to the actuator, as shown in (2). The data face packet dropout and random time delay in the controller-to-actuator links. It means the data packets of the controller may reach the actuator or may drop due to network traffic. This communication is modeled with random events as

$$
\begin{cases}\text { Event 1: } & \text { Succesful transmission and } u(k) \text { is available, } \\ \text { Event 2: } & \text { Link fail and } u(k) \text { is not available. }\end{cases}
$$

For the random events that occur in the controller-toactuator links, we introduce $b(k)$ that is a binary random vector. $b_{i}(k)=1$ indicates that the packet delivered successfully in the $i$ th channel while $b_{i}(k)=0$ indicates that the packet dropped. Here, $i \in\{1,2, \ldots, m\}$. We assume the following probabilities for $b_{i}(k)$ :

$$
\begin{aligned}
& \operatorname{Pr}\left(b_{i}(k)=1\right)=E\left[b_{i}(k)\right]=\bar{b}_{i}(k), \\
& \operatorname{Pr}\left(b_{i}(k)=0\right)=1-\bar{b}_{i}(k),
\end{aligned}
$$

where $\operatorname{Pr}\left\{b_{i}(k)=1\right\}$ is the probability of packet transmission in $\mathrm{C} / \mathrm{A}$ links and $E$ is the mathematical expectation. When the probability of $b_{i}(k)=1$, it means maximum probability or successful transmission of data packet through C/A links, else the packet is lost.

The data packets that successfully transmit via C/A link face random time delays $\tau_{k c a}$. We assume that $\tau_{k c a}$ varies randomly between 0 and $\tau_{2}$, where $\tau_{2}$ is the maximum time delay. We model the time delays $\tau_{k c a}$ with a Markov chain $\Theta_{2}$ with $N_{2}$ number of stats, i.e., $\Theta_{2} \in\left\{1,2, \ldots, N_{2}\right\}$. Let $\rho_{2}$ be the transition probability matrix of the Markov chain that is given as

$$
\rho_{2}=\left[\rho_{i_{2} j_{2}}\right],
$$

where $\quad \rho_{i_{2} j_{2}}=\mathbb{P}\left(\Theta=j_{2} \mid \Theta=i_{2}\right)=\mathbb{P}\left(\tau_{k c a+1}=j_{2} \mid \tau_{k c a}=\right.$ $\left.i_{2}\right) \geq 0$, for all $i_{2}, j_{2} \in \Gamma_{2}$ and $\sum_{j_{2}=0}^{\tau_{1}} \rho_{i_{2} j_{2}}=1, \rho_{i_{2} j_{2}} \geq 0$. The transition probability matrix is similar to the sensor-tocontroller link. We assume that $\rho=\rho_{1}+\rho_{2}$.

The industrial surveillance system may experience local faults in the actuators. The effects of actuators' faults can be modeled as

$$
\Theta=\operatorname{diag}\left(\theta_{1}, \theta_{2}, \ldots, \theta_{m}\right)
$$

where $\Theta$ denotes the faults matrix and functions $\theta_{l} \in[0,1]$, for $l=1, \ldots, m_{2}$, indicate the status of each individual actuator, that is,

$$
\theta_{l}= \begin{cases}1, & \text { the actuator is working normally, } \\ 0, & \text { the actuator is failed completely, } \\ \text { otherwise, } & \text { the actuator is partially working. }\end{cases}
$$

The plant's input $u^{*}(k)$ with the communication link impurities can be written as

$$
u^{*}(k)=\Theta \overline{b_{i}}(k) u\left(k-\tau_{k c a}\right)+g(\cdot)
$$

where $g(\cdot)$ is the nonlinear term that may add due to technical limitations [18].

Remark 2. Equation (16) demonstrates that the control signal may or may not reach the actuator. If $\bar{b}_{i}(k)=1$, then the delayed version of $u(k)$ with time delay of $\tau_{k c a}$ will be available to the actuator. The packet dropouts and time delays in $\mathrm{C} / \mathrm{A}$ links and S/C links are considered independent random events. We consider time delays for those packets which are successfully transmitted via network links. The lump delay is $\tau_{k}=\tau_{k s c}+\tau_{k c a}$.

Consequently, considering the augmented state vector as $\eta(k)=\left[x^{T}(k), x^{T}(k-1), x^{T}(k-2), \ldots, x^{T}\left(k-\tau_{k}-1\right), x^{T}\right.$ 
$\left.\left(k-\tau_{k}\right)\right]^{T} \in R^{\left(\tau_{k+1}\right) n}$. Substituting (10) and (16) in (1) leads us to the closed-loop dynamics of the networked system as follows:

$$
\begin{aligned}
\eta(k+1)= & \left(A+B \Theta \overline{b_{i}}(k) F\left(\tau_{k}\right) \Omega \overline{a_{i}}(k) C\right) \eta(k) \\
& +B \Theta \overline{b_{i}}(k) F\left(\tau_{k}\right) f(\cdot)+B g(\cdot) .
\end{aligned}
$$

Remark 3. The output feedback-based closed-loop networked system derived in (17) is a nonlinear stochastic system with two data packet dropout modes $\overline{a_{i}}(k)$ and $\overline{b_{i}}(k)$ and two time-delay modes $\tau_{k s c}$ and $\tau_{k c a}$.

The system in (17) can be written in a compact form as follows:

$$
\eta(k+1)=\bar{A} \eta(k)+\widehat{f}(\cdot)+\widehat{g}(\cdot)
$$

where

$$
\begin{aligned}
& \bar{A}=\left[\begin{array}{cccccc}
A & 0 & 0 & \cdots & 0 & B \Theta \overline{b_{i}}(k) F\left(\tau_{k}\right) \Omega \overline{a_{i}}(k) C \\
I & 0 & 0 & \cdots & 0 & 0 \\
0 & I & 0 & \cdots & 0 & 0 \\
\vdots & \vdots & \ddots & \cdots & \vdots & \vdots \\
0 & 0 & 0 & \cdots & I & 0
\end{array}\right] \\
& \widehat{f}(\cdot)=\left[\begin{array}{c}
B \Theta \overline{b_{i}}(k) F\left(\tau_{k}\right) f(\cdot) \\
0 \\
0 \\
\vdots \\
0
\end{array}\right] \\
& \widehat{g}(\cdot)=\left[\begin{array}{c}
B g(\cdot) \\
0 \\
0 \\
\vdots \\
0
\end{array}\right]
\end{aligned}
$$

\section{Fault-Tolerant Control Design}

Assumption 1. We assume that there is no packet dropouts and time delays in the bounded nonlinear functions that we add to the sensor and actuator faults $f(y(k))$ and $g(u(k))$, respectively, which satisfy the following local Lipschitz conditions:

$$
\begin{gathered}
f^{T}(y(k)) f(y(k)) \leq v^{2} y^{T}(k) y(k), \\
g^{T}(y(k)) g(u(k)) \leq \gamma^{2} y^{T}(k) u(k),
\end{gathered}
$$

where $v>0$ and $\gamma>0$ are the bounding parameters for nonlinear functions $f(y(k))$ and $g(u(k))$.

Definition 1. The Markovian jump nonlinear stochastic system in (18) is robust stochastic stable with any initial condition $\left(\eta(0), \tau_{k 0}, \overline{a_{i}}(0), \overline{b_{i}}(0)\right)$ if

$$
E\left\{\sum_{k=0}^{\infty}\left\|\eta_{k}\right\|^{2} \mid \eta(0), \tau_{k} 0, a_{i}(0), b_{i}(0)\right\} \leq \infty
$$

where $\eta_{k}(0)$ denotes the solution of (18) at time $k$ under the initial conditions $\left(\zeta(0), \tau_{k 0}, a_{i}(0)\right.$, and $\left.b_{i}(0)\right)$.

Here, we present our LMI for the proposed remotely located output feedback controller design to ensure stochastic stability.

Theorem 1. Consider the closed-loop system for the industrial surveillance systems as in (18), and let $\nu>0, \gamma>0, \epsilon_{1}>0$, and $\epsilon_{2}>0$ be the given scalars. A desired output feedback controller gain of form (10) exists if there exist matrices $X_{i}>0$, $R_{i}, G_{i}, Q_{i}$, and $M_{i}\left(\tau_{k}\right)$, satisfying

$$
\left[\begin{array}{cccccccc}
\xi_{11} & 0 & 0 & \xi_{14} & \xi_{15} & \xi_{16} & \xi_{17} & \xi_{18} \\
\star & -\epsilon_{1} I & 0 & \xi_{24} & \xi_{25} & \xi_{26} & 0 & 0 \\
\star & \star & -\epsilon_{2} I & \xi_{34} & \xi_{35} & \xi_{36} & 0 & 0 \\
\star & \xi_{42} & \xi_{43} & \xi_{44} & 0 & 0 & 0 & 0 \\
\xi_{51} & \star & \star & 0 & \xi_{55} & 0 & 0 & 0 \\
\xi_{61} & \star & \star & 0 & 0 & \xi_{66} & 0 & 0 \\
\star & \star & \star & \star & \star & \star & -\bar{\Lambda}_{1} & 0 \\
\star & \star & \star & \star & \star & \star & \star & -\bar{\Lambda}_{2} I
\end{array}\right]<0,
$$

where $\xi_{11}=-G_{i}^{T} X_{i}^{-1} G_{i}, \quad \xi_{44}=\xi_{55}=\xi_{66}=\xi_{99}=$ diag $\left\{X_{k_{1}^{i}}, \ldots,-X_{k_{m}^{i}},-X_{j}\right\}$,

$$
\begin{aligned}
& \xi_{14}=\xi_{15}=\xi_{16}=\left\{\begin{array}{c}
\sqrt{\rho_{i} k_{1}^{i}}\left\{\bar{A} G_{i}\right\}^{T} \\
\vdots \\
\sqrt{\rho_{i} k_{m}^{i}}\left\{\bar{A} G_{i}\right\}^{T} \\
\sqrt{1-\rho_{k}^{i}}\left\{\bar{A} G_{i}\right\}^{T}
\end{array}\right\}, \\
& \xi_{24}=\xi_{25}=\xi_{26}=\left\{\begin{array}{c}
\sqrt{\rho_{i} k_{1}^{i}}\left\{M^{T}\left(\tau_{k}\right)\right\} \\
\vdots \\
\sqrt{\rho_{i} k_{m}^{i}}\left\{M^{T}\left(\tau_{k}\right)\right\} \\
\sqrt{1-\rho_{k}^{i}}\left\{M^{T}\left(\tau_{k}\right)\right\}
\end{array}\right\} \text {, } \\
& \xi_{34}=\xi_{35}=\xi_{36}=\left\{\begin{array}{c}
\sqrt{\rho_{i} k_{1}^{i}} \bar{B}^{T} \\
\vdots \\
\sqrt{\rho_{i} k_{m}^{i}} \bar{B}^{T} \\
\sqrt{1-\rho_{k}^{i}} \bar{B}^{T}
\end{array}\right\} \text {, } \\
& \xi_{51}=\xi_{61}=\left\{\begin{array}{c}
\sqrt{\rho_{i} k_{1}^{i}} \bar{A}^{T} \\
\vdots \\
\sqrt{\rho_{i} k_{m}^{i}} \bar{A}^{T} \\
\sqrt{1-\rho_{k}^{i}} \bar{A}^{T}
\end{array}\right\} \text {, }
\end{aligned}
$$




$$
\begin{gathered}
\xi_{42}=\left\{\begin{array}{c}
\sqrt{\rho_{i} k_{1}^{i}}\left\{M\left(\tau_{k}\right) G_{i}\right\} \\
\vdots \\
\sqrt{\rho_{i} k_{m}^{i}}\left\{M\left(\tau_{k}\right) G_{i}\right\} \\
\sqrt{1-\rho_{k}^{i}}\left\{M\left(\tau_{k}\right) G_{i}\right\}
\end{array}\right\}, \\
\xi_{43}=\left\{\begin{array}{c}
\sqrt{\rho_{i} k_{1}^{i}}\left\{\bar{B} G_{i}\right\} \\
\vdots \\
\sqrt{\rho_{i} k_{m}^{i}}\left\{\bar{B} G_{i}\right\} \\
\sqrt{1-\rho_{k}^{i}}\left\{\bar{B} G_{i}\right\}
\end{array}\right\}, \\
\xi_{49}=\left\{\begin{array}{c}
\sqrt{\rho_{i} k_{1}^{i}}\{\bar{H}\} \\
\vdots \\
\sqrt{\rho_{i} k_{m}^{i}}\{\bar{H}\} \\
\sqrt{1-\rho_{k}^{i}}\{\bar{H}\}
\end{array}\right\},
\end{gathered}
$$$$
\bar{\Lambda}_{1}=\left(\epsilon_{1} v^{2}\right)^{-1} I \bar{\Lambda}_{2}=\left(\epsilon_{2} \gamma^{2}\right)^{-1} I \text {. According to the definition }
$$$$
F i\left(\tau_{k}\right) \bar{C} G_{i}=R_{i} \bar{C} \text {, it is derived that }
$$$$
F i\left(\tau_{k}\right)=R_{i} Q^{-1} \text {. }
$$

Proof. We choose the Lyapunov function candidate as

$$
\begin{aligned}
V\left(\tau_{k}, a_{i}(k), b_{i}(k)\right)= & \eta^{T}(k) P_{i}\left(\tau_{k}, a_{i}(k), b_{i}(k)\right) \eta(k), \\
E\left[\Delta V\left(\tau_{k}, a_{i}(k), b_{i}(k)\right)\right]= & E\left[\eta^{T}(k+1)\left\{\sum_{k=0}^{N} \rho_{i j} P_{j}\left(\tau_{k+1}, a_{i}(k+1), b_{i}(k+1)\right)\right\} \eta(k+1)\right] \\
& -\eta^{T}(k) P_{i}\left(\tau_{k}, a_{i}(k), b_{i}(k)\right) \eta(k), \\
\overline{P_{j}}= & \left\{\sum_{k=0}^{N} \rho_{i j} P_{j}\left(\tau_{k+1}, \bar{a}(k+1), \bar{b}(k+1)\right)\right\},
\end{aligned}
$$

and then, we have

$$
\begin{aligned}
E\left[\Delta V\left(\tau_{k}, a_{i}(k), b_{i}(k)\right)\right] \leq & \eta^{T}(k)\left\{\left(A+B \Theta \overline{b_{i}}(k) F\left(\tau_{k}\right) \Omega \overline{a_{i}}(k) C\right)^{T} \overline{P_{j}}(\ldots)\right\} \eta(k) \\
& +\eta^{T}(k)\left\{\left(A+B \Theta \overline{b_{i}}(k) F\left(\tau_{k}\right) \Omega \overline{a_{i}}(k) C\right)^{T} \overline{P_{j}} B \Theta \overline{b_{i}}(k) F\left(\tau_{k}\right)\right\} f(\cdot)+\eta^{T}(k) \\
& \left\{\left(A+B \Theta \overline{b_{i}}(k) F\left(\tau_{k}\right) \Omega \overline{a_{i}}(k) C\right)^{T} \overline{P_{j}} B\right\} g(\cdot)+f^{T}(\cdot)\left\{B \Theta \overline{b_{i}}(k) F\left(\tau_{k}\right)\right\}^{T} \\
& \overline{P_{j}}\left(A+B \Theta \overline{b_{i}}(k) F\left(\tau_{k}\right) \Omega \overline{a_{i}}(k) C\right) \eta(k)+f^{T}(\cdot)\left\{B \Theta \overline{b_{i}}(k) F\left(\tau_{k}\right)\right\}^{T} \overline{P_{j}}(\ldots) \\
& +f^{T}(\cdot)\left\{B \Theta \overline{b_{i}}(k) F\left(\tau_{k}\right)\right\}^{T} \overline{P_{j}} B g(\cdot)+g^{T}(\cdot) B^{T} \overline{P_{j}}\left(A+B \Theta \overline{b_{i}}(k) F\left(\tau_{k}\right) \Omega \overline{a_{i}}(k) C\right) \eta(k) \\
& +g^{T}(\cdot) B^{T} \overline{P_{j}}\left\{B \Theta \overline{b_{i}}(k) F\left(\tau_{k}\right)\right\} f(\cdot)+g^{T}(\cdot) B^{T} \overline{P_{j}}(\ldots)-\varepsilon_{1} f^{T}(\cdot) f(\cdot)+\varepsilon_{1} \nu^{2} y^{T}(k) y(k) \\
& +\varepsilon_{2} \gamma^{2} u^{T}(k) u(k)-\varepsilon_{2} g^{T}(\cdot) g(\cdot)-\zeta^{T}(k) P_{i} \zeta(k) .
\end{aligned}
$$

It can be shown that $\Delta V \leq 0$ implies $E\left\{\sum_{k=0}^{\infty} \| \eta\right.$ $\left.k \|^{2} \mid \eta(0), \tau_{k 0}, a_{(0)}, b_{I}(0)\right\} \leq \infty$ that guaranteed stochastic stability for the augmented state vector given in (18). By defining the augmented state vectors,

$$
\xi=\left[\begin{array}{c}
\eta(k) \\
f(\cdot) \\
g(\cdot)
\end{array}\right],
$$

we can write equation (26) as 


$$
\xi_{0}=\left[\begin{array}{ccc}
\xi_{11}-\lambda & \xi_{12} & \xi_{13} \\
\xi_{21} & \xi_{22}-\epsilon_{1} I+\left(\epsilon_{2} \gamma^{2} F\left(\tau_{k}\right)\right)^{T}(\ldots) & \xi_{23} \\
\xi_{31} & \xi_{32} & \xi_{33}-\epsilon_{2} I
\end{array}\right],
$$

$\xi_{11}=\bar{A}^{T} \overline{P_{j} A}$, where $\bar{A}=\left(A+B \Theta \overline{b_{i}}(k) F\left(\tau_{k}\right) \Omega \overline{a_{i}}(k) C\right), \lambda=$ $\bar{C} \epsilon_{1} \nu^{2} \bar{C}+\left(\epsilon_{2} \gamma^{2} F\left(\tau_{k}\right) \Omega \alpha(k) \bar{C}\right)^{T}(\ldots)-\zeta^{T}(k) P_{i} \zeta(k)$, and $\xi_{12}=\bar{A}^{T} \overline{P_{j}} M\left(\tau_{k}\right)$, where $M\left(\tau_{k}\right)=\bar{B} \Theta \beta(k), \quad \xi_{13}=\bar{A}^{T} \overline{P_{j} B}$, where $\bar{B}=B, \quad \xi_{21}=M\left(\tau_{k}\right)^{T} \overline{P_{j} A}, \quad \xi_{22}=M\left(\tau_{k}\right)^{T} \overline{P_{j}} M\left(\tau_{k}\right)$, $\xi_{23}=M\left(\tau_{k}\right)^{T} \overline{P_{j} B}, \quad \xi_{31}=\bar{B}^{T} \overline{P_{j} A}, \quad \xi_{32}=\bar{B}^{T} \overline{P_{j}} M\left(\tau_{k}\right)$, and $\xi_{33}=\bar{B}^{T} \overline{P_{j} B}$.

Multiplication of $\operatorname{diag}\left(G_{i}^{T}, I\right)$ from left and $\operatorname{diag}\left(G_{i}, I\right)$ from right to $\xi_{0}$ and defining $X_{i}=P_{i}^{-1}, F\left(\tau_{k}\right) \bar{C} G_{i}=R_{i} \bar{C}$, then, by using the Schur complement and the introduction of network-induced uncertainties as in [18] results the following is obtained:

$$
\left[\begin{array}{cccccccc}
\xi_{11} & 0 & 0 & \xi_{14} & \xi_{15} & \xi_{16} & \xi_{17} & \xi_{18} \\
\star & -\epsilon_{1} I & 0 & \xi_{24} & \xi_{25} & \xi_{26} & 0 & 0 \\
\star & \star & -\epsilon_{2} I & \xi_{34} & \xi_{35} & \xi_{36} & 0 & 0 \\
\star & \xi_{42} & \xi_{43} & \xi_{44} & 0 & 0 & 0 & 0 \\
\xi_{51} & \star & \star & 0 & \xi_{55} & 0 & 0 & 0 \\
\xi_{61} & \star & \star & 0 & 0 & \xi_{66} & 0 & 0 \\
\star & \star & \star & \star & \star & \star & -\bar{\Lambda}_{1} & 0 \\
\star & \star & \star & \star & \star & \star & \star & -\bar{\Lambda}_{2} I
\end{array}\right]<0,
$$

where $\xi_{11}=-G_{i}^{T} X_{i}^{-1} G_{i}$,

$$
\begin{aligned}
& \xi_{44}=\xi_{55}=\xi_{66}=\operatorname{diag}\left\{X_{k_{1}^{i}}, \ldots,-X_{k_{m}^{i}},-X_{j}\right\}, \\
& \xi_{14}=\xi_{15}=\xi_{16}=\left\{\begin{array}{c}
\sqrt{\rho_{i} k_{1}^{i}}\left\{A+M\left(\tau_{k}\right) R_{i} \bar{C}\right\}^{T} \\
\vdots \\
\left.\sqrt{\rho_{i} k_{m}^{i}}\left\{A+M\left(\tau_{k}\right) R_{i} \bar{C}\right\}^{T}\right\} \\
\sqrt{1-\rho_{k}^{i}}\left\{A+M\left(\tau_{k}\right) R_{i} \bar{C}\right\}^{T}
\end{array}\right\}, \\
& \xi_{24}=\xi_{25}=\xi_{26}=\left\{\begin{array}{c}
\sqrt{\rho_{i} k_{1}^{i}}\left\{M^{T}\left(\tau_{k}\right)\right\} \\
\vdots \\
\sqrt{\rho_{i} k_{m}^{i}}\left\{M^{T}\left(\tau_{k}\right)\right\} \\
\sqrt{1-\rho_{k}^{i}}\left\{M^{T}\left(\tau_{k}\right)\right\}
\end{array}\right\}, \\
& \xi_{34}=\xi_{35}=\xi_{36}=\left\{\begin{array}{c}
\sqrt{\rho_{i} k_{1}^{i}} \bar{B}^{T} \\
\vdots \\
\sqrt{\rho_{i} k_{m}^{i}} \bar{B}^{T} \\
\sqrt{1-\rho_{k}^{i}} \bar{B}^{T}
\end{array}\right\},
\end{aligned}
$$

$$
\begin{aligned}
& \xi_{51}=\xi_{61}=\left\{\begin{array}{c}
\sqrt{\rho_{i} k_{1}^{i}}\left\{A+M\left(\tau_{k}\right) R_{i} \bar{C}\right\}^{T} \\
\vdots \\
\sqrt{\rho_{i} k_{m}^{i}}\left\{A+M\left(\tau_{k}\right) R_{i} \bar{C}\right\}^{T} \\
\sqrt{1-\rho_{k}^{i}}\left\{A+M\left(\tau_{k}\right) R_{i} \bar{C}\right\}^{T}
\end{array}\right\} \\
& \xi_{42}=\left\{\begin{array}{c}
\sqrt{\rho_{i} k_{1}^{i}}\left\{M\left(\tau_{k}\right) G_{i}\right\} \\
\vdots \\
\sqrt{\rho_{i} k_{m}^{i}}\left\{M\left(\tau_{k}\right) G_{i}\right\} \\
\sqrt{1-\rho_{k}^{i}}\left\{M\left(\tau_{k}\right) G_{i}\right\}
\end{array}\right\}, \\
& \xi_{43}=\xi_{17}=\left\{\begin{array}{c}
\sqrt{\rho_{i} k_{1}^{i}}\left\{\bar{B} G_{i}\right\} \\
\vdots \\
\sqrt{\rho_{i} k_{m}^{i}}\left\{\bar{B} G_{i}\right\} \\
\sqrt{1-\rho_{k}^{i}}\left\{\bar{B} G_{i}\right\}
\end{array}\right\}, \\
& \xi_{18}=\left\{\begin{array}{c}
\sqrt{\rho_{i} k_{1}^{i}}\{\bar{B}\} \\
\vdots \\
\sqrt{\rho_{i} k_{m}^{i}}\{\bar{B}\} \\
\sqrt{1-\rho_{k}^{i}}\{\bar{B}\}
\end{array}\right\},
\end{aligned}
$$

$\bar{\Lambda}_{1}=\left(\epsilon_{1} v^{2}\right)^{-1} I \bar{\Lambda}_{2}=\left(\epsilon_{2} \gamma^{2}\right)^{-1} I$. According to the definition $F i\left(\tau_{k}\right) \bar{C} G_{i}=R_{i} \bar{C}$, it is derived that

$$
F i\left(\tau_{k}\right)=R_{i} Q^{-1} .
$$

Lemma 2. For $\bar{C}$ with rank $p$, there exist nonsingular matrices $Q_{i}$ such that $Q_{i} \bar{C}=\bar{C} G_{i}$ for which there exist a full-rank matrices $G_{i}$ satisfying

$$
G_{i}=\zeta\left[\begin{array}{cc}
G_{i 1} & 0 \\
G_{i 2} & G_{i 3}
\end{array}\right] \zeta^{T}=\zeta_{i 1} G_{i 1} \zeta_{i 1}^{T}+\zeta_{i 2} G_{i 2} \zeta_{i 1}^{T}+\zeta_{i 2} G_{i 3} \zeta_{i 2}^{T},
$$

where $Q_{i}=U^{T} \sigma G_{i 1}^{-1} \sigma^{-1} U$. Here, $U$ is find by singular value decomposition of matrix $\bar{C}$ that is $\bar{C}=U \bar{C} \zeta_{i}=U \bar{C}\left[\begin{array}{ll}\zeta_{i 1} & \zeta_{i 2}\end{array}\right]=U \bar{C}\left[\begin{array}{ll}\sigma & 0\end{array}\right]$, where $\sigma$ is the nonzero singular value of $\bar{C}$.

Remark 4. The output matrix $\bar{C}$ is assumed as a full-row rank. The bilinear matrix inequalities are transformed under this assumption into a set of linear matrix inequalities. The assumption is removed in [10]. As it can be satisfied for many practical applications, that is, why it is not a major constrain. In [18], a similar problem is solved for the faulttolerant control design of networked systems with the state feedback control design. The state feedback control is not significant for real-time applications due to the rare accessibility of all states. 


\section{Simulation Study}

A gimbal-based camera sensor communicates with a remote controller through a communication link and the control controls the actuator over a network link, as shown in Figure 2. State variables of the surveillance system are the angular positions $\phi$ and $\psi$ of inner and outer channels and angular velocities of inner and outer channels $\dot{\phi}$ and $\dot{\psi}$ of the gimbal system, respectively. To actuate the system, two brushless DC motors are used with torques $U_{\tau_{1}}, U_{\tau_{2}}$, respectively. Angular velocity of the inner channel, which holds the camera sensor, is taken as output of the surveillance system. The system dynamics $[23,25]$ for the surveillance system with initial conditions are

$$
\begin{aligned}
{\left[\begin{array}{c}
\dot{\phi} \\
\dot{\psi} \\
\ddot{\phi} \\
\ddot{\psi}
\end{array}\right] } & =\left[\begin{array}{cccc}
0 & 1 & 0 & 0 \\
8.96 & 0 & 0 & 2.43 \\
0 & 0 & 0 & 1 \\
2.06 & 0 & 2.4 & 0.2
\end{array}\right]\left[\begin{array}{l}
\phi \\
\psi \\
\dot{\phi} \\
\dot{\psi}
\end{array}\right]+\left[\begin{array}{cc}
0 & 0 \\
0.15 & 0 \\
0 & 0 \\
0 & 11.3
\end{array}\right]\left[\begin{array}{l}
U_{\tau_{1}} \\
U_{\tau_{2}}
\end{array}\right], \\
C & =\left[\begin{array}{llll}
0 & 0 & 1 & 0 \\
0 & 0 & 0 & 0
\end{array}\right] .
\end{aligned}
$$

There are two actuators and one sensor. The first actuator is working normally and the second actuator and the sensor both are working partially. The fault matrices are given as follows: $\Theta=\left[\begin{array}{cc}1 & 0 \\ 0 & 0.7\end{array}\right]$ and $\Omega=[0.6]$. The nonlinearities that may add to faults are $g(\cdot)=0.6 u(k) \cos (u(k))$ and $f(\cdot)=0.7 y(k) \cos (y(k))$. The complex transition probability matrices for the sensor-to-controller link and the controller-to-actuator link, respectively, are given as

$$
\begin{aligned}
& \rho_{1}=\left[\begin{array}{cccc}
0.3 & 0.4 & ? & 0.3 \\
? & 0.8 & 0.8 & ? \\
0.5 & 0.1 & ? & 0.7 \\
0.9 & ? & 0.5 & ?
\end{array}\right], \\
& \rho_{2}=\left[\begin{array}{cccc}
0.3 & 0.4 & ? & 0.3 \\
? & 0.7 & 0.4 & ? \\
0.5 & 0.6 & ? & 0.7 \\
0.1 & ? & 0.5 & ?
\end{array}\right],
\end{aligned}
$$

if $\tau_{k c a}=\tau_{k s c}=3$.

The Bernoulli stochastic variables for sensor-to-controller and controller-to-actuator links are $\overline{a_{i}}(k)$ $=\overline{b_{i}}(k)=0.7$. The data packet dropouts and random time delay for the sensor-to-controller link and controller-to- actuator links are given in Figures 3 and 4, respectively. Similarly, the Markov evaluation of the controller modes and the system modes are given in Figure 5.

The simulation results of a smart surveillance system for the tolerance of simultaneous local sensors and actuator faults propagated effects of faults of other nodes, and network-induced uncertainties are shown in Figures 6 to 8. In Figure 6, we plot the tolerance of faults with and without the nonlinear effects. The blue solid line is for tracking without nonlinear effects and the red dotted line is for tracking with nonlinearities. Similarly, Figure 7 demonstrates the tracking performance with and without network-induced uncertainties. For comparison in tracking response and fault tolerance, we plot the output of a conventional controller and the proposed controller in presence of network-induced uncertainties without faults in Figure 8. Oscillation in the tracking response of the conventional controller will further increase if we add faults, especially, if we add simultaneous faults. This proves the superiority of the proposed controller in fault tolerance and in tracking targets. In Figure 9, we plot the tracking response with states to show that the states are stable even in the presence of the sensor fault. Starting with different initial conditions, all the states are stable and converge to zero with output tracking the reference input. The output feedback controller gain of the remote controller for the industrial smart surveillance system are calculated using MATLAB LMI toolbox and is given as

$F 1=\left[\begin{array}{c}-0.2169 \\ -0.5682 \\ 0.0217 \\ 0.0284\end{array}\right]$,

$F 2=\left[\begin{array}{c}-0.9865 \\ -2.5846 \\ 0.0987 \\ 0.1292\end{array}\right]$,

$F 3=\left[\begin{array}{c}0.7612 \\ -1.9943 \\ 0.0761 \\ 0.0997\end{array}\right]$, 


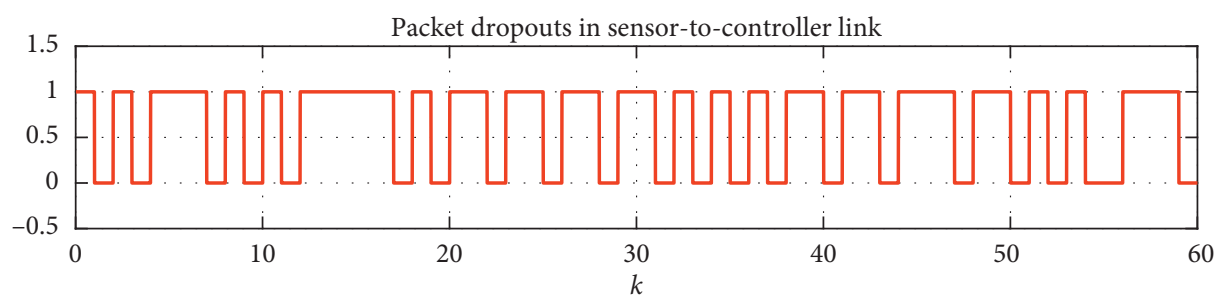

$-\mathrm{P}_{\mathrm{r}}\left(a_{i}=0.7\right)$

(a)

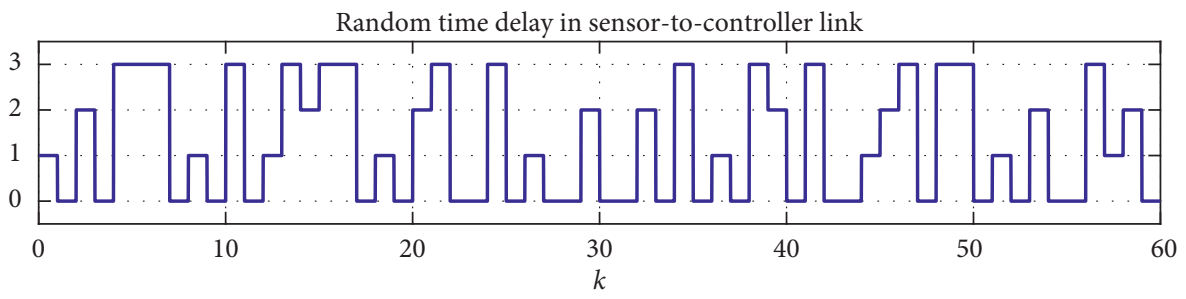

$-\tau_{1}=3$

(b)

Figure 3: Data packet dropouts and random time delays in the sensor-to-controller communication link.

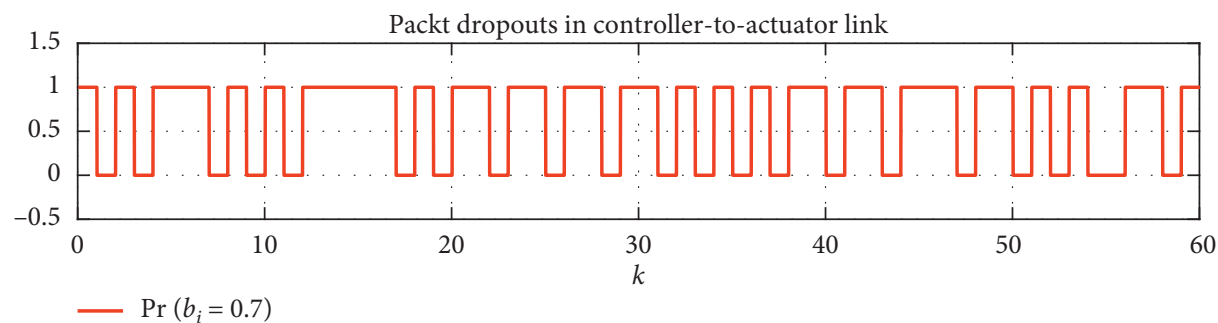

(a)

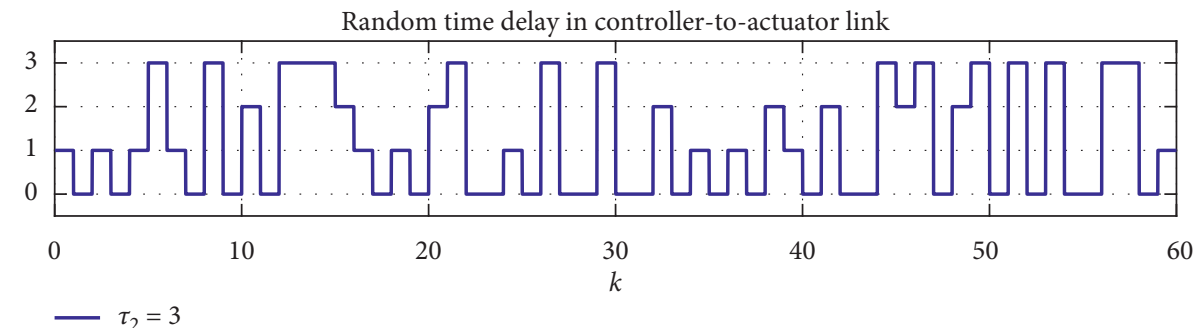

(b)

FIgURE 4: Data packet dropouts and random time delays in the controller-to-actuator communication link. 


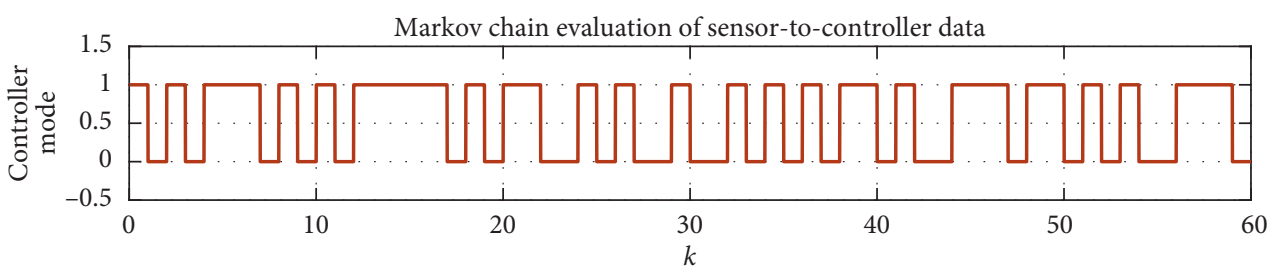

(a)

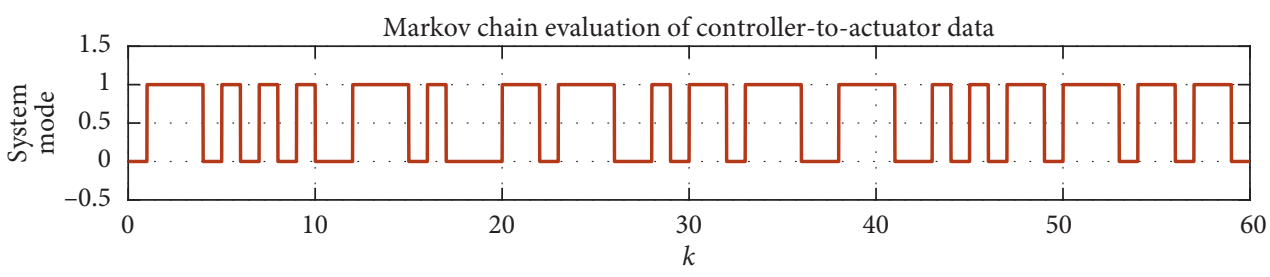

(b)

Figure 5: Markov chain evaluation of controller and system modes.

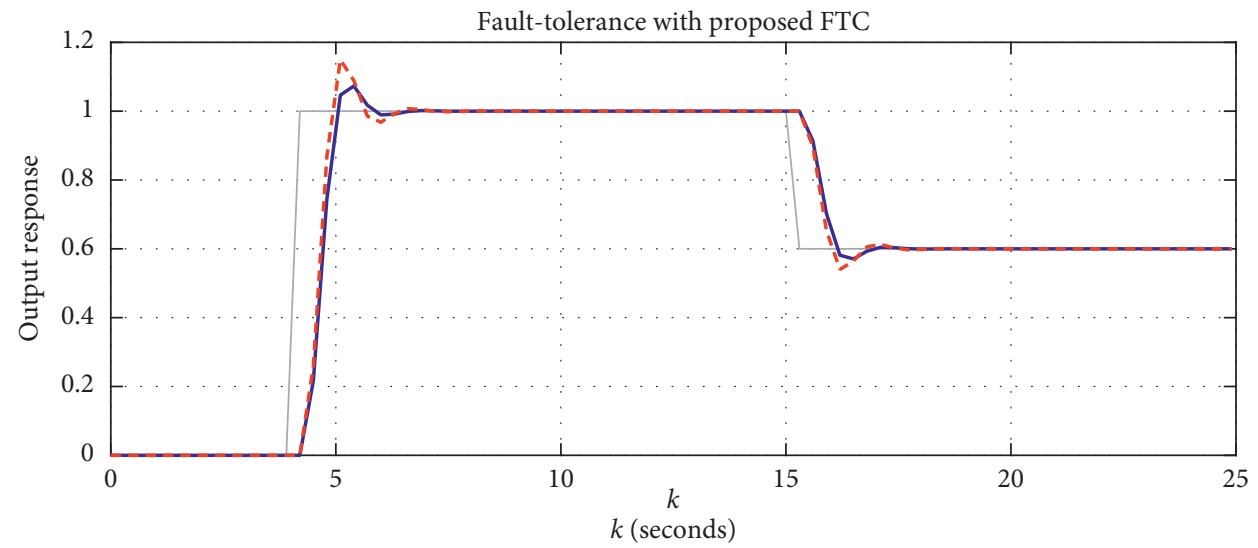

Tracking without uncertainties - - - Tracking with uncertainties

FIGURE 6: Tracking performance with and without nonlinearities.

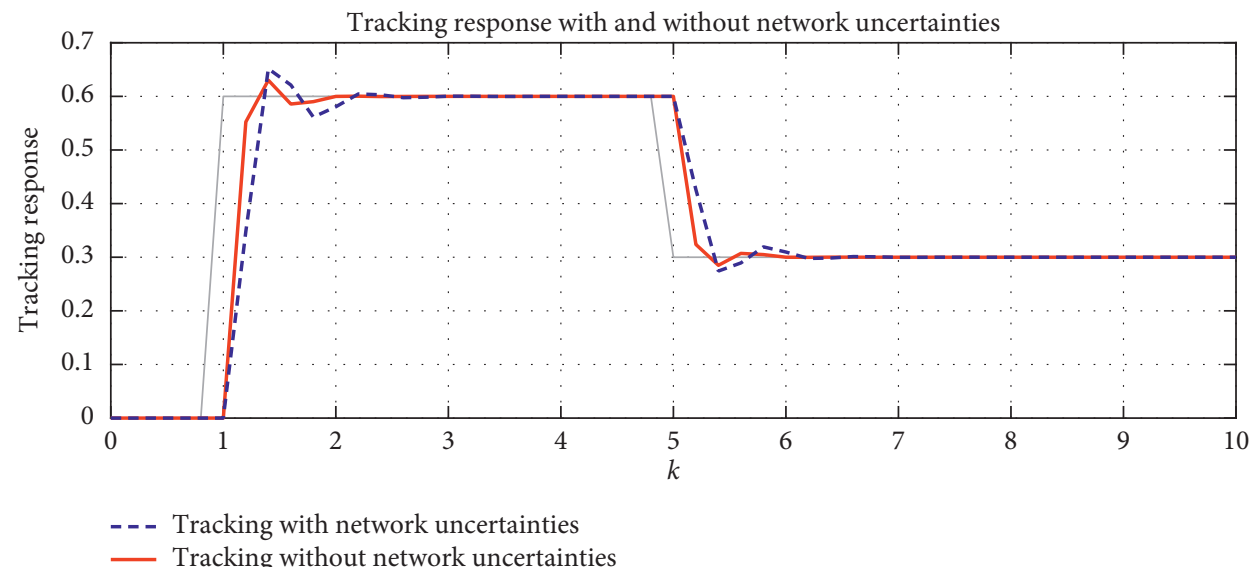

Figure 7: Tracking response with and without network-induced uncertainties. 


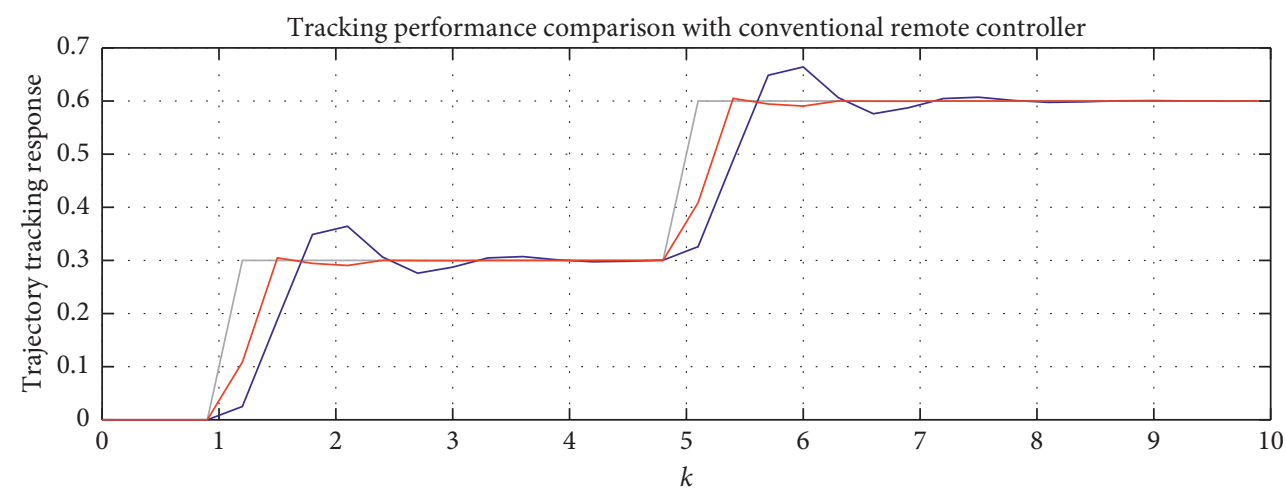

_ Tracking with conventional remote controller

_ Tracking with proposed remote controller

FIGURE 8: Tracking response comparison with the conventional remote controller without faults.

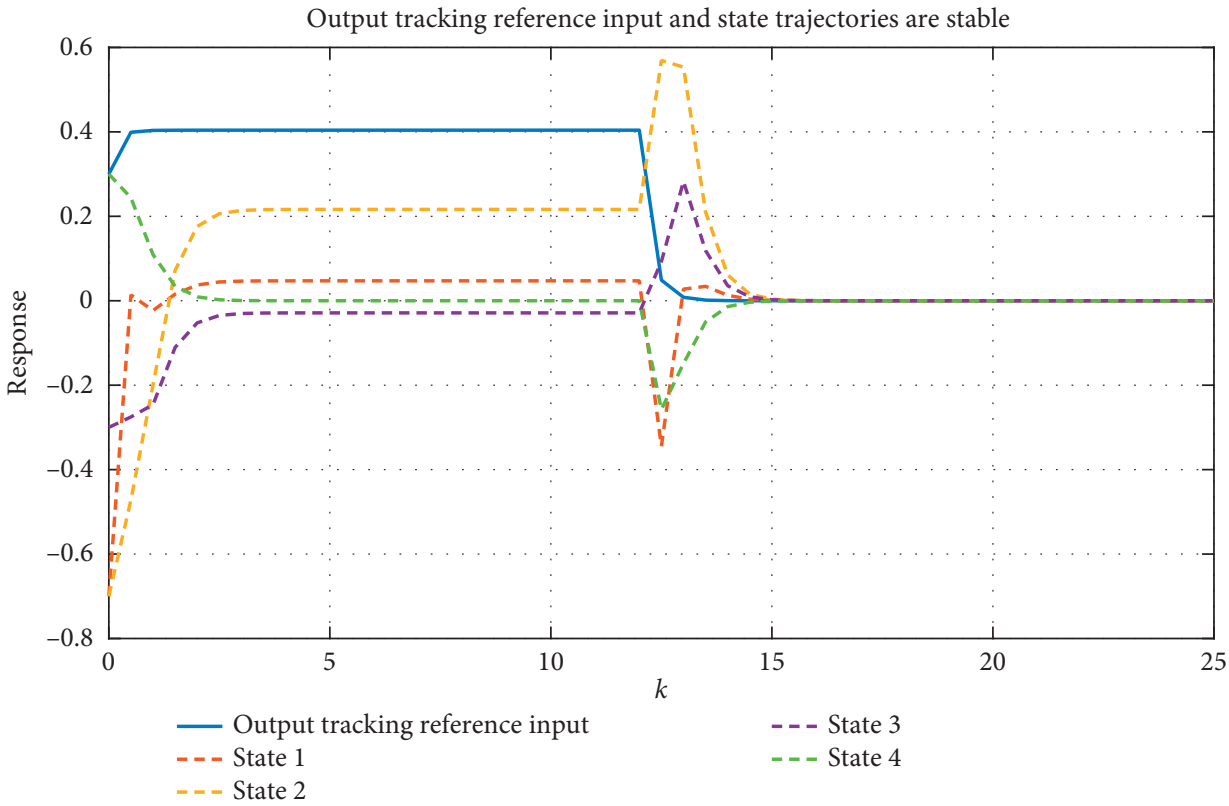

FIGURE 9: Tracking performance with stable state response in the presence of sensor and actuator simultaneous faults.

\section{Conclusion}

We presented two new fault models for sensors and actuators that are more general than the existing fault models. Random time delays and uncertain data packet dropouts of the network links are modeled in S/C links and C/A network lines separately by using Markov chains and Bernoulli processes, respectively. The remote fault-tolerant controller successfully tracks the reference input and tolerates the effects of simultaneous faults in the presence of networkinduced uncertainties. Simulation results for the surveillance system verify the stochastic stability of the systems and the applicability of the controller design.

\section{Data Availability}

The data used to support the findings of this study are included within the article.

\section{Conflicts of Interest}

The authors declare that they have no conflicts of interest.

\section{Acknowledgments}

This research was supported by Taif University Researchers Supporting Project number (TURSP-2020/144), Taif University, Taif, Saudi Arabia.

\section{References}

[1] L. D. Xu, E. L. Xu, and L. Li, "Industry 4.0: state of the art and future trends," International Journal of Production Research, vol. 56, no. 8, pp. 2941-2962, 2018.

[2] H. Zhang, Y. Shi, J. Wang, and H. Chen, "A new delay-compensation scheme for networked control systems in controller area networks," IEEE Transactions on Industrial Electronics, vol. 65, no. 9, pp. 7239-7247, 2018. 
[3] T. Zou, S. Wu, and R. Zhang, "Improved state space model predictive fault-tolerant control for injection molding batch processes with partial actuator faults using GA optimization," ISA Transactions, vol. 73, pp. 147-153, 2018.

[4] X. Li and X.-B. Wu, "Guaranteed cost fault-tolerant controller design of networked control systems under variable-period sampling," Information Technology Journal, vol. 8, no. 4, pp. 537-543, 2009.

[5] Q. Zhu, K. Lu, and Y. Zhu, "Observer-based feedback control of networked control systems with delays and packet dropouts," Journal of Dynamic Systems, Measurement, and Control, vol. 138, no. 2, pp. 1-8, 2016.

[6] Y. Li, H. Li, and W. Sun, "Event-triggered control for robust set stabilization of logical control networks," Automatica, vol. 95, pp. 556-560, 2018.

[7] M. Bahreini and J. Zarei, "Robust finite-time stabilization for networked control systems via static output-feedback control: markovian jump systems approach," Circuits, Systems, and Signal Processing, vol. 37, no. 4, pp. 1523-1541, 2018.

[8] L. Zhang, T. Yang, P. Shi, and Y. Zhu, Analysis and Design of Markov Jump Systems with Complex Transition Probabilities, Springer, Berlin, Germany, 2016.

[9] M. F. Braga, C. F. Morais, R. C. Oliveira, and P. L. Peres, "Robust stability and stabilization of discrete-time markov jump linear systems with partly unknown transition probability matrix," in Proceedings of the American Control Conference (ACC), pp. 6784-6789, Washington, DC, USA, June 2013.

[10] X. Liu, M. R. P. Reddy, and P. S. Ge, "Dynamic output feedback asynchronous control of networked markovian jump systems," IEEE Transactions on Systems, Man, and Cybernetics: Systems, vol. 50, no. 7, pp. 2705-2715, 2020.

[11] C. Tan, L. Li, and H. Zhang, "Stabilization of networked control systems with both network-induced delay and packet dropout," Automatica, vol. 59, pp. 194-199, 2015.

[12] W. Sun, Q. Li, C. Zhao, and S.-K. Nguang, "Mode-dependent dynamic output feedback $H \infty$ control of networked systems with markovian jump delay via generalized integral inequalities," Information Sciences, vol. 520, pp. 105-116, 2020.

[13] M. Bahreini and J. Zarei, "Static-output feedback controller design for a class of nonlinear networked control systems with random delay and data packet dropout," Systems Science \& Control Engineering, vol. 4, no. 1, pp. 192-198, 2016.

[14] A. A. Amin and K. M. Hasan, "A review of fault tolerant control systems: advancements and applications," Measurement, vol. 143, pp. 58-68, 2019.

[15] X. Guo, Y. Tan, F. Wang, Y. Tan, and F. Wang, "Modeling and fault propagation analysis of cyber-physical power system," Energies, vol. 13, no. 3, p. 539, 2020.

[16] Y.-J. Zhang, L.-S. Hu, and L.-S. Hu, "Fault propagation inference based on a graph neural network for steam turbine systems," Energies, vol. 14, no. 2, p. 309, 2021.

[17] T. Wang, X. Wei, T. Huang et al., "Modeling fault propagation paths in power systems: a new framework based on event SNP systems with neurotransmitter concentration," IEEE Access, vol. 7, pp. 12798-12808, 2019.

[18] M. Bahreini and J. Zarei, "Robust fault-tolerant control for networked control systems subject to random delays via static-output feedback," ISA Transactions, vol. 86, pp. 153$162,2019$.

[19] A. S. Khan, A. Q. Khan, N. Iqbal et al., "Distributed fault detection and isolation in second order networked systems in a cyber-physical environment," ISA Transactions, vol. 103, pp. 131-142, 2020.
[20] M. Nazir, A. Q. Khan, G. Mustafa, and M. Abid, "Robust fault detection for wind turbines using reference model-based approach," Journal of King Saud University-Engineering Sciences, vol. 29, no. 3, pp. 244-252, 2017.

[21] Q. Zhu, K. Lu, and Y. Zhu, " $H_{\infty}$ guaranteed cost fault-tolerant control of double-fault networked control systems: piecewise delay method," Mathematical Problems in Engineering, vol. 2019, Article ID 6348727, 19 pages, 2019.

[22] A. Balasubramanyam, B. N. Pavan Kumar, Y. H. Chai, and A. K. Patil, "Inspired by human eye: vestibular ocular reflex based gimbal camera movement to minimize viewpoint changes," Symmetry, vol. 11, p. 101, 2019.

[23] A. Mahmood, A. Q. Khan, G. Mustafa et al., "Gimbal based robotic eye for dynamic social environment," in Proceedings of the 2020 International Symposium on Recent Advances in Electrical Engineering and Computer Sciences (RAEE and CS), Islamabad, Pakistan, October 2020.

[24] B. Ekstrand, "Equations of motion for a two-axes gimbal system," IEEE Transactions on Aerospace and Electronic Systems, vol. 37, no. 3, pp. 1083-1091, 2001.

[25] R. Cunha, M. Malaca, and V. Sampaio, "Gimbal control for vision-based target tracking," in Proceedings of the European Conference on Signal Processing, Coruña, Spain, September 2019. 